\title{
Limited thoracoplasty in the management of complicated pulmonary aspergillomas
}

\author{
M AL-ZEERAH, K JEYASINGHAM \\ From the Department of Thoracic Surgery, Frenchay Hospital, Bristol
}

ABSTRACT Complicated pulmonary aspergilloma is a life threatening condition. Indications for surgery are few and the procedures conservative. Three patients were successfully treated with limited thoracoplasty. Limited thoracoplasty in combination with other conservative measures may provide effective palliation in selected cases.

\section{Introduction}

Pulmonary manifestations of infestation with Aspergillus have been classified' as saprophytic (aspergilloma), allergic, and septicaemic or pyaemic (invasive). The treatment of the last two types is nonsurgical, but controversy exists over the role of surgery in the management of aspergilloma $\mathrm{a}^{2-4}$ because of the high incidence of postoperative complications. ${ }^{5}$ Most surgeons advocate resection only in the presence of a complication, such as haemorrhage or sepsis though when sepsis is the main problem nonresectional procedures may be of value. ${ }^{27} \mathrm{We}$ report three patients with complicated aspergillomas treated by resection or cavernostomy with a limited thoracoplasty.

\section{Case reports}

\section{PATIENT 1}

A 56 year old woman presented with episodes of haemoptysis complicating recurrent chest infections; one haemoptysis was considered to be massive. Ten years previously she had undergone a right sided posterolateral thoracotomy and partial (apical) pleurectomy after suture obliteration of a leaking bulla causing recurrent pneumothoraces. Three years later she developed increasing shortness of breath, a productive cough, and weight loss. Radiologically there was a thick walled cavity in the right apex. Despite being sputum negative for acid fast organisms, she received a full course of "insurance chemotherapy." A chest radiograph at her present admission

\footnotetext{
Address for reprint requests: Mr K Jeyasingham, Department of
} Thoracic Surgery, Frenchay Hospital, Bristol BS16 1LE.

Accepted 21 September 1989 confirmed the cavity at the right apex and conventional tomography showed an opacity within the cavity (figs $1 a$ and $1 b$ ). Bronchoscopy excluded a proximal obstruction to the upper lobe bronchus or its segments. Aspergillus fumigatus was found in the lavage aspirates. She underwent resection of a completely destroyed right upper lobe and an adjacent apical segment of the lower lobe. The remaining lung was infiltrated by fibrous tissue and was stuck to the parietal chest wall. A limited (five rib) apical thoracoplasty was completed to obliterate the residual space. When last seen two years after surgery, she was no longer troubled by her chest and was leading a relatively normal life (fig $1 c$ ).

\section{PATIENT 2}

A 54 year old male storekeeper presented with increasing shortness of breath and several episodes of haemoptysis. He had been under observation with chronic obstructive airways disease for several years and his chest radiograph had consistently shown an opacity within a cystic area in the right upper zone (fig $2 a$ ). He was positive for aspergillus precipitins. A chest radiograph at admission showed a large opacity in the right upper zone with a fluid level (fig $2 b$ ). He had purulent sputum with a raised peripheral white cell count $\left(21 \times 10^{9} / 1\right)$. An intercostal tube drained frank pus and his general condition improved. Pus and air continued to drain through the intercostal tube and he underwent a right sided posterolateral thoracotomy with a limited thoracoplasty to remove the $2 \mathrm{nd}$, 3rd, and 4th ribs; apicolysis was completed with drainage of the cavity. He was discharged home with minimal drainage from the cavernostomy and with complete obliteration of the bronchopleural fistula. At follow up six months later he was still short of breath but had no discharge from the sinus tract and a chest radiograph showed obliteration of the apical space. 


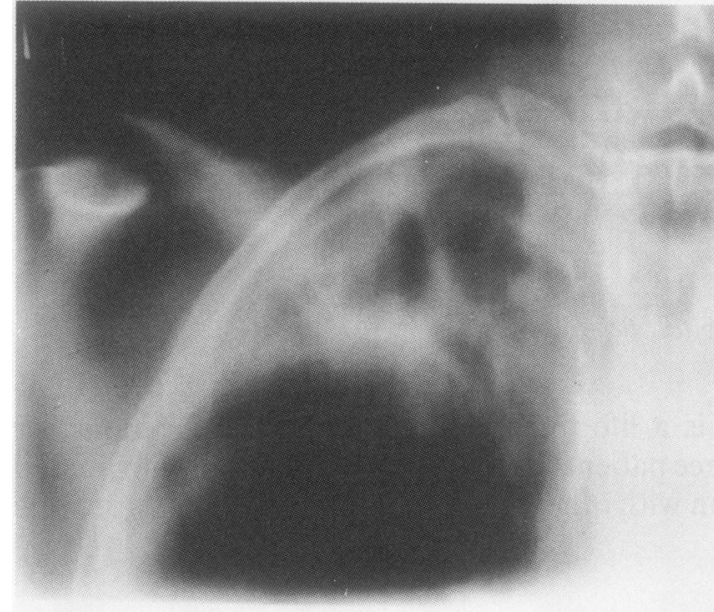

(a)

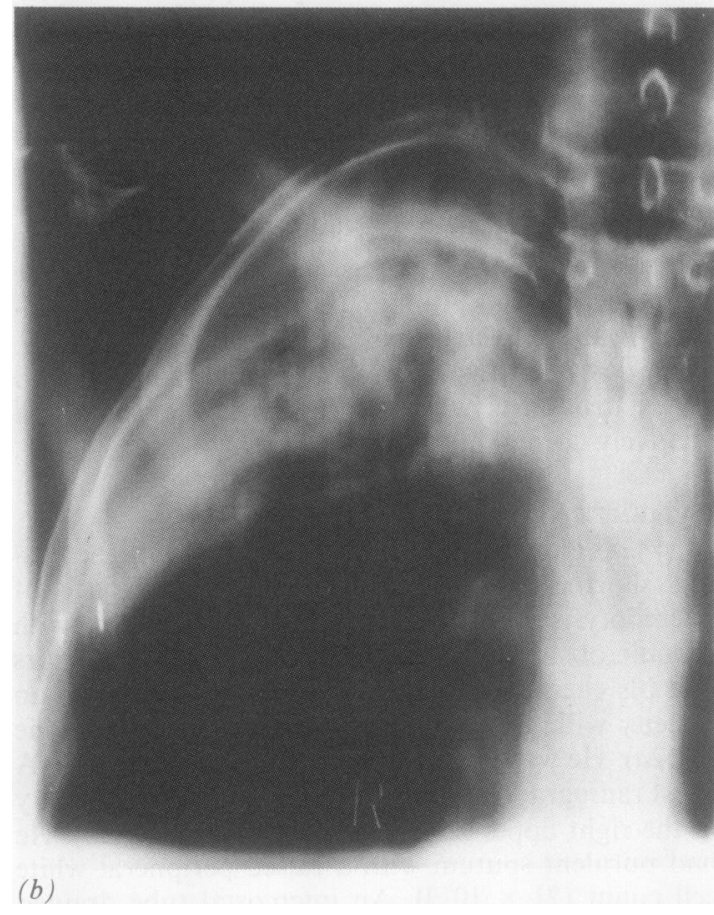

PATIENT 3

A 39 year old woman had undergone a right sided upper lobectomy at the age of 11 years for a congenital lung cyst. This was complicated by stump breakdown and infection resulting in a bronchopleural fistula. She later developed a chronic space infection with $A$ fumigatus, and became positive for aspergillus precipitins. Radiologically there was progressive deterioration of both lung fields (fig 3). She was maintained on bronchodilators and corticosteroids. In view of rapidly deteriorating lung function she had a

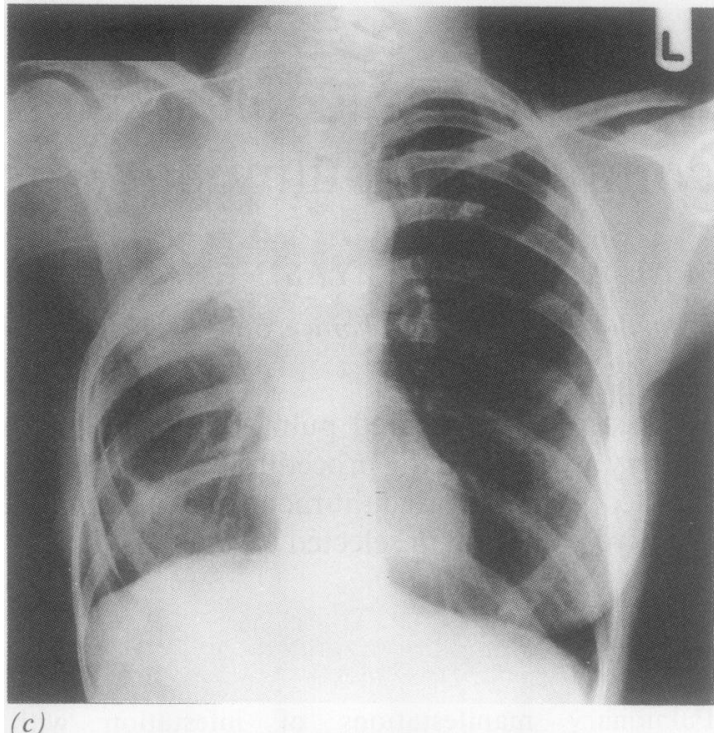

Fig 1 ( $a$ and $b$ ) Tomograms of the right apex showing $a$ pre-existing cavity in 1982 and a larger cavity containing a ball of fungal hyphae in 1986. (c) Follow up chest radiograph after an apical thoracoplasty removing the $2 \mathrm{nd}, 3 \mathrm{rd}, 4 \mathrm{th}$, and Sth ribs and stripping the periosteum off the under surface of the 1st rib with the apicolysis. Note that the right shoulder has not dropped because the 1 st rib has been retained. Despit the radiological configuration of the rib cage the cosmetic appearance of the shoulder and chest is maintained.

cavernostomy and a limited thoracoplasty. At operation the 2nd, 3rd, and 4th ribs were removed, apico- $\overline{0}$ lysis was completed, and the cavity was drained. She 3 required intensive postoperative care but was discharged home with minimal drainage from her cavernostomy site. At follow up six months later she was less short of breath and the drainage site had healed. $A$ 응 chest radiograph showed considerable clearing in both $\times$ lung fields.

\section{Discussion}

The prevalence of aspergilloma in patients with a known tuberculosis cavity is extremely high, $90 \%$ ․ㅡ․ occurring in previously known cavities. ${ }^{4}$ The most $N$ common symptom in aspergilloma is haemoptysis, usually minor recurrent episodes associated with 0 infective exacerbations but occasionally massive $\mathcal{W}$ haemoptysis endangering the patient's life. The minor episodes can be adequately managed with bed rest, 0 antibiotics, postural drainage, and humidified oxygen, $\mathbb{D}$ but major bleeding usually warrants urgent surgical $\stackrel{?}{-}$ intervention; ${ }^{5}$ embolisation of the bronchial arteries $\frac{0}{0}$ preoperatively may reduce the risk of surgery. ${ }^{8}$ About $\frac{\vec{D}}{\mathbb{D}}$ $30 \%$ of patients with recurrent minor haemoptyses 

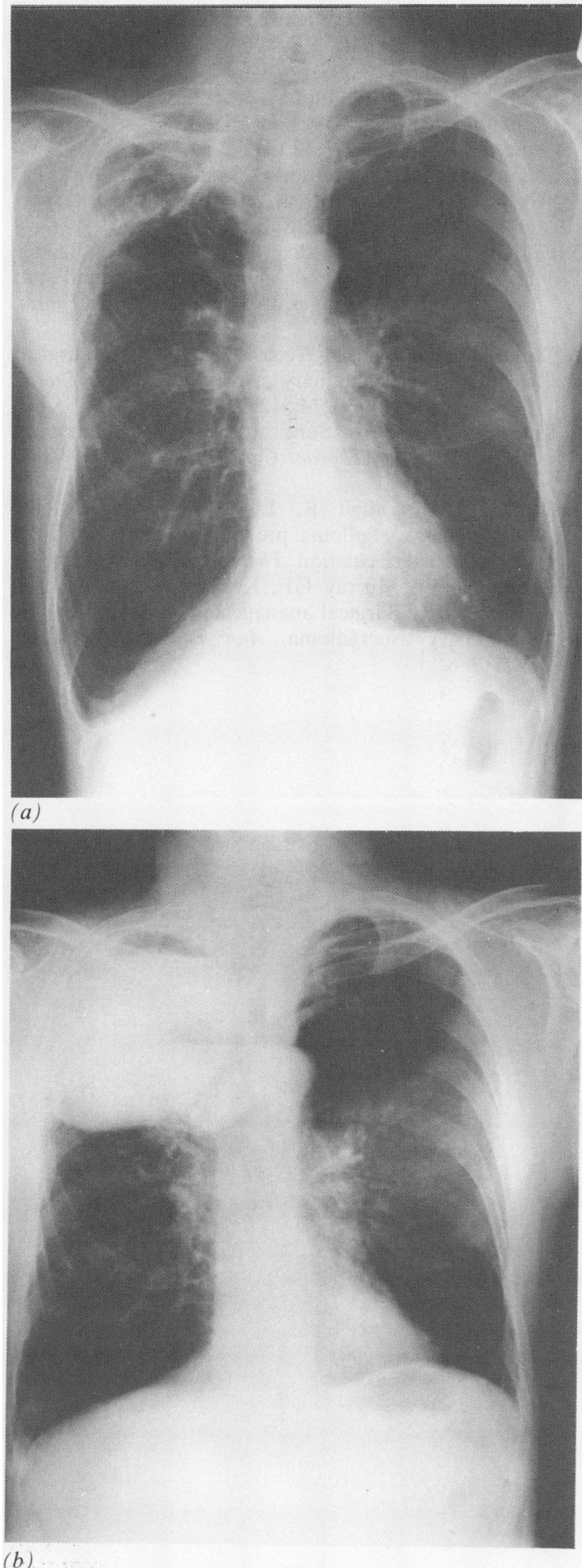

Fig 2 (a) Radiograph of the chest showing a cavity in the right apex with an aspergilloma. (b) The cavity seen in (a), now enlarged and filled with fluid and air.

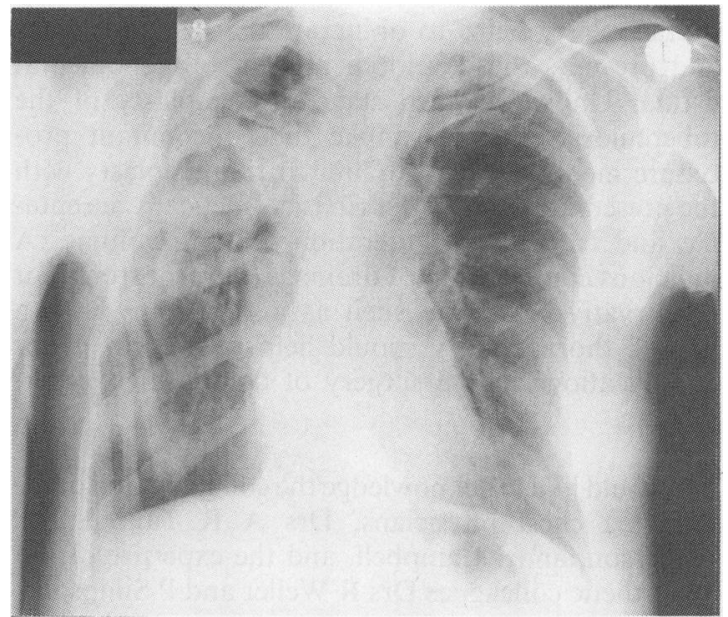

Fig 3 Preoperative radiograph of the chest showing an aspergilloma in the right apical cavity and diffuse lung disease due to invasive aspergillosis.

proceed to a major bleed, ${ }^{6 y}$ supporting the view held by some that any degree of haemoptysis in a patient with an aspergilloma should be an indication for surgery. ${ }^{9}$ Haemorrhage remains the main indication for surgery, but sepsis and malignant change may also necessitate surgical intervention.

Various surgical procedures, mostly developed in the days of surgery for tuberculosis before the advent of antituberculous chemotherapy, have been reported. Resection procedures in the presence of previous chronic lung disease are fraught with complications, such as persistent air leak, bronchopleural fistula, and space infection ${ }^{7}$ resulting from the non-expansion of the fibrotic residual lung. Technically the procedures are complicated and difficult because of the extensive induration and fibrosis. As a result of the poor general health of the patients and their immunocompromised state the postoperative period is often complicated by infection. Consequently the mortality of such procedures has been as high as $34 \% .^{2}$ Lesser procedures such as cavernostomy, thoracoplasty, and muscle transposition have therefore gained popularity as either an initial or a concomitant procedure. Cavernostomy establishes adequate drainage and allows irrigation, but has the disadvantage of establishing a bronchopleurocutaneous fistula. Muscle transposition and limited thoracoplasty both help to obliterate any cavities and to obliterate an established bronchopleural fistula when used as an initial procedure, and they help to close a bronchopleurocutaneous fistula that results from cavernostomy when used as a secondary procedure. Daly et $a l^{2}$ reported three cases where thoracoplasty was used as a concomitant procedure to overcome complications following primary resection, and another report of thoracoplasty was in a patient with poor pulmonary reserve unsuitable for resection. ${ }^{7}$ When combined with resection limited 
thoracoplasty helps to obliterate the resulting space and prevents the development of a bronchopleural fistula. Unlike the two stage thoracoplasty of the tuberculosis era, and unlike the concomitant procedure mentioned above, limited thoracoplasty with the preservation of the 1st rib is cosmetically acceptable and causes little alteration in lung volume. A judicious combination of drainage (cavernostomy) or conservative resection such as a lobectomy with a limited thoracoplasty should help to prevent major complications in the surgery of complicated aspergilloma.

We would like to acknowledge the collaboration of the referring chest physicians, Drs A R Tanser, J P Anderson, and I Campbell, and the expertise of our anaesthetic colleagues Drs R Weller and P Simpson.

\section{References}

1 Hinson KFW, Moon AJ, Plummer NS. Bronchopulmonary aspergillosis. Thorax 1952;7:317-33.
2 Daly RC, Pairolero PC, Piehler JM, Trastek VF, Spencer Payne W, Berenatz PE. Pulmonary aspergilloma. $J$ Thorac Cardiovasc Surg 1986;92:981-8.

3 Karas A, Hankins JR, Attar S, Miller JE, McLaughlin JS Pulmonary aspergillosis: an analysis of 41 patients. Ann Thorac Surg 1976;22:1-7.

4 Soltanzadeh MD, Sychulis MD, Sadr F, Bolanowski PJ, Neville WE. Surgical treatment of pulmonary aspergilloma. Ann Surg 1977;186:13-6.

5 Faulkner SL, Vernon R, Brown PB, Fisher RD, Bender HW. Hemoptysis and pulmonary aspergilloma: operative versus nonoperative treatment. Ann Thorac Surg 1978;25:389-92.

6 Garvey J, Crastnopol P, Weisz D, Khan F. The surgical treatment of pulmonary aspergillomas. $J$ Thorac Cardiovasc Surg 1977;74:542-7.

7 Saab S, Almond C. Surgical aspects of pulmonary aspergillosis. J Thorac Cardiovasc Surg 1974;68: $455-60$.

8 Hughes CF, Waugh R, Lindsay D. Surgery for pulmonary aspergilloma: preoperative embolisation of the bronchial circulation. Thorax 1986;41:324-5.

9 Battaglini JW, Murray GF, Keagy BA, Starek PJK, Wilcox BR. Surgical management of symptomatic pulmonary aspergilloma. Ann Thorac Surg 1985; 39:512-6. 